\title{
Application of Compounding Feedforward PID Algorithm in the Positioning Control of Computerized Flat Knitter
}

\author{
Haipeng Pan, Xiaoyan Feng, Minming Gu \\ Institute of Automation \\ Zhejiang Sci-Tech University \\ Hangzhou, 310018, China
}

\begin{abstract}
In allusion to the problem of low positioning precision in the handpiece positioning control of computer knitting machine, this paper applies the compound feedforward PID control algorithm to the position control system, combines with 51 Micro Control Unit (MCU) to realize the high-precision positioning control. In contrast the traditional algorithm PID control technology, this system simulates on the MATLAB platform. The results show that this algorithm has a good following performance and less error, will be helpful for improving the positioning accuracy, meanwhile with a high practical value.
\end{abstract}

Keywords-Computerized flat knitter, Compounding feedforward PID algorithm, MCU

\section{INTRODUCTION}

Computerized flat knitting machine is a kind of Dualneedle bed warp knitting machine; it is mainly used in fabric manufacturing of woolen or cashmere sweater [1]. In the computerized flat knitting machine, driven mechanism of handpiece is one of the most important equipments; its accurate movement is the key of fabric's quality. Most flat knitting machines usually use common PID control algorithm, but the position has great error, and affects the quality of the fabric. This paper bases on the researches of the operating principle of shogging system and the technological requirements, adopts servo motors, drivers, photoelectric encoders and Microcontrollers to build adjustment system of motor speed. The compounding feedforward PID algorithm is used in the handpiece location's control system, which raises the precision and rapidity of locating control.

\section{THE PRINCIPLED AND STRUCTURE OF THE SYSTEM}

Shogging mechanism of flat knitting machine is also called Shaker or Bed-moving, which adopts the mode that front of the needle bed to shog, the back of needle bed to fix. The transmission mechanism drives the handpiece working with needle selection and the looping elements to weave. The main transmission is driven by a servo motor; transmitted through two-stage synchronous belts; than the handpiece is driven by synchronous belt to do a shogging reciprocating movement [2]. When the knitter begins the sliding weaving, the servo motor pulls the handpiece quickly to finish the repeated movements on the condition that the motor owns the high controlling accuracy, high response speed and large output torque. So, in order to guarantee the control accuracy, this paper designs the system which used Micro Control Unit (MCU) as its control core, is driven by the AC servo based on the compounding feedforward control. The drivers can sample the feedback of motor encoder directly. AC servo system is consisted of both position-loop and speed-loop, which greatly improves the performance. The results of the controller are used in flat knitting machine show that: AC servo system accelerates from 0 to $2000 \mathrm{r} / \mathrm{min}$ uses only a few milliseconds, ideal for this controlling application.

This paper designs the hardware structure of control system is shown in Figure. 1. The keyboard is used to set input value and edited parameters; LCD shows the monitoring data. Photoelectrical encoders are mounted on the spindle motor, to achieve the detection of motor speed; servo motor is drove the transmission mechanism of flat knitting machine, so these constitute a low-cost automatic control system.

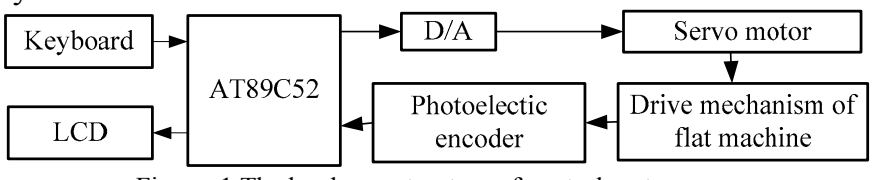

Figure .1 The hardware structure of control system

AC servo control system can generally realize three control models include velocity control model, torque control model and position control model [3], using position control mode in the shogging control. The block diagram of the shogging closed-loop control is shown in Figure.2. The setting value and the feedback data from the photoelectric encoder, which would be calculated by the AT89C52 and transmitted to the servo motor through the D/A transformation, then the servo motor controls the knitter shogging mechanism, accomplishes the position positioning action.

\section{THE DESIGN OF SHOGGING POSITION CONTROLLER}

\section{A. The analysis of shogging mechanism of flat knitting machine}

In this paper, the position control of handpiece uses servo motor as its drive unit. The conveyor belts drive the handpiece to shogging left and right. According to the different kinds of the clothing tissue, the knitter should constantly change the location of needle bed to get the 
correct location through front and back needle. Meanwhile the knitter adds and reduces the number of needles to change the textile fabric's width. If the knitter cannot ensure the location accuracy, as a result the knitting needle will be destroyed. So headpiece's position can be controlled more accuracy, which will achieve the knitting process requirements.

The basic requirement of servo system to the position regulator is as follow: on the premise of ensuring the positioning control accuracy and without overshoot, the system has a dynamic response as fast as possible, realizes the minimum position tracking error and the shortest position setting time [4]. After testing, the velocity curve of needle bed's shogging mechanism is shown in Figure.3.

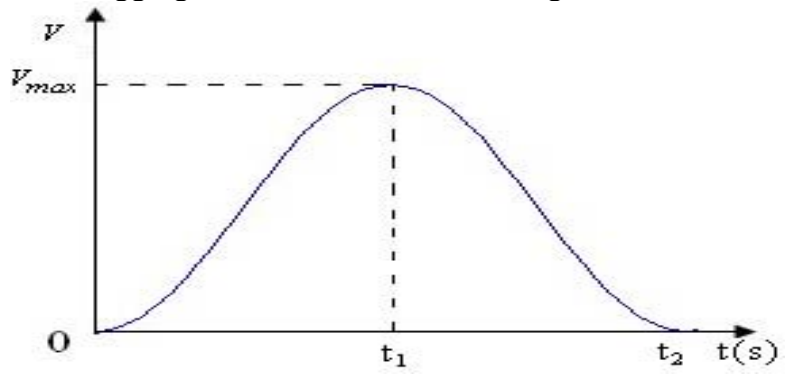

Figure. 3 The velocity curve of needle bed's shogging mechanism

When the computer knitting machine working, the handpiece is doing constantly shogging, the speed raises from $0-V \max -0$. The whole shogging time is $\mathrm{T}$, at the moment of 0 or $\mathrm{t} 2$, the handpiece has the max acceleration. If we need the handpiece to finish the designated shogging distance in the shortest time, the handpiece should get the high speed, meanwhile it requires the servo motor to provide the large acceleration, and the speed formula of handpiece is shown as follow:

$$
v=n_{D} \times C \times \frac{\pi d}{60 \times 1000}
$$

$\mathrm{v}(\mathrm{m} / \mathrm{s})$ is the speed of handpiece, $\mathrm{nD}(\mathrm{r} / \mathrm{min})$ is the servo motor speed; $C$ is gear ratio, $\mathrm{d}(\mathrm{mm})$ is the driving pulley diameter. In the formula (1) the $\mathrm{C}$ and $\mathrm{d}$ are the constants. So the moving speed of the handpiece depends on the servo motor speed.

This paper uses the M method [5] to test the motor speed that is nd $(\mathrm{r} / \mathrm{s})$.

$$
n_{d}=\frac{m}{N \cdot t}
$$

$\mathrm{N}$ is the number of pulses when motor has a whirl, $\mathrm{m}$ is the number of pulses which is output by the rotary encoder in a certain time. If there is one pulse, the corresponding movement of the handpiece is $0.01 \mathrm{~mm}$, when there are $\mathrm{m}$ pulses in a certain time, the shogging movement is $h$ $(\mathrm{mm})=0.01 \cdot \mathrm{m}$, we get the number of the pulses are $\mathrm{m}=\mathrm{h} / 0.01$, we calculate the formula (2), and the result is shown as formula (3).

$$
h=\frac{N \cdot t \cdot n d}{100}
$$

We conclude that the accuracy of the servo motor speed determinate on the accuracy of the shogging movement. So we should improve the controlling accuracy of the whole servo system. Meanwhile the common PID controller cannot achieve the high accuracy, on the other sides, the compounding feedforward control can raise the tracking performance and controlling accuracy of the shogging movement.

\section{B. The thought of compounding feedforward control PID algorithm}

Feedforward control is designed in the classic control theory basic on the compounding control idea. The openloop feedforward control is lead out directly from the given sign, together with the close-loop feedback control, constitutes a compounding control system [6]. The structure of the system is shown in Figure.4.

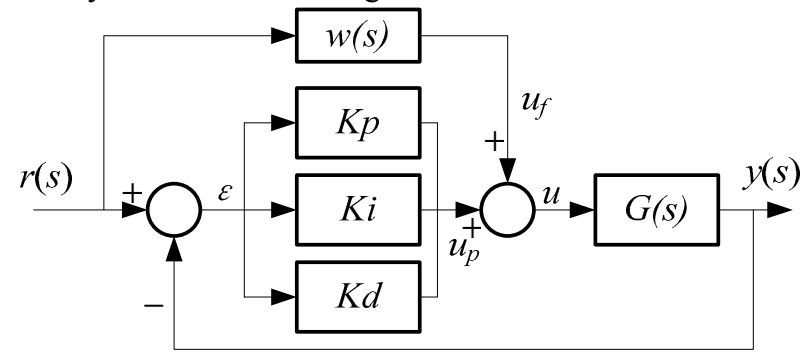

Figure.4 The structure of the compounding feedforward control PID

In Figure $.4 \mathrm{r}(\mathrm{s})$ is input value, $\mathrm{y}(\mathrm{s})$ is output value, $\varepsilon$ is system error, w(s) is a feedforward compensator, $\mathrm{Kp}, \mathrm{Ki}, \mathrm{Kd}$ constitute a PID controller (its function is $\mathrm{G0}(\mathrm{s}), \mathrm{Kp}$ is scale factor, Ti is integral coefficient, Td is differential coefficient), $\mathrm{G}(\mathrm{s})$ is a control object. The close-loop transfers' function can be expressed as:

$$
\frac{y(s)}{r(s)}=\frac{G(s) G_{0}(s)+w(s) \cdot G(s)}{1+G(s) G_{0}(s)}
$$

If $y(s)=r(s)$, the output of actual location in an ideal state should equal to input of the given location, so:

$$
\frac{G(s) G_{0}(s)+w(s) \cdot G(s)}{1+G(s) G_{0}(s)}=1
$$
follow:

The transfer functions of feedforward compensator as

$$
w(s)=\frac{1}{G(s)}
$$

The function of control output as follow:

$$
u_{f}(s)=r(s) \frac{1}{G(s)}
$$


The function of total control output can be calculated by:

$$
\begin{aligned}
& u(t)=u_{p}(t)+u_{f}(t) \\
& =K_{p}\left[e(t)+\frac{1}{T_{i}} \int_{0}^{T} e(t) d t+T_{d} \frac{d e(t)}{d t}\right]+u_{f}(t)
\end{aligned}
$$

\section{MATLAB SIMULATION AND RESULTS ANALYSES}

This paper adopts the servo motor rated speed is $2000 \mathrm{r} / \mathrm{min}$. When the motor has a whirl, the number of pulse is 2000 , the sampling time is $1 \mathrm{~ms}$. After modeling the shogging control operation of textile machine, the system achieves the parameters $\mathrm{h}=0.6667 \mathrm{~mm}$, $\alpha_{0}=1.26, \alpha_{1}=0.0081, \alpha_{2}=0.00067$. This paper uses $\mathrm{M}$ documentation to compile algorithm programs on the MATLAB platform. $\mathrm{Kp}, \mathrm{Ki}, \mathrm{Kd}$ are optimized by the experimental test method in the programs, meanwhile transmitted the optimal value to the MCU, then the system obtains the best simulation results. Figure. 5 is the transfer function's diagram of shogging system.

$\mathrm{Gl}(\mathrm{s})$ is the transfer function of AC servo control, its mathematical model [7] is shown as follow:

$$
G_{1}(s)=\frac{K}{s\left(\alpha_{2} s^{2}+\alpha_{1} s+\alpha_{0}\right)}
$$

$\mathrm{G} 2$ (s) is the value of shogging movement, according to the modeling parameters to calculate the transfer functions of the generalized object in the shogging control shows as follow:

$$
G(s)=G_{1}(s) \cdot G_{2}(s)=\frac{65}{s\left(0.00067 s^{2}+0.0081 s+1.26\right)}
$$

The input sign is $y(s)=-0.5 \cos (6 \pi t)$. When the parameters are $\mathrm{Kp}=1.795, \mathrm{Ki}=1.76, \mathrm{Kd}=0.0395$, the results are best. Simulation results are shown in Figure.6 and Figure.7.
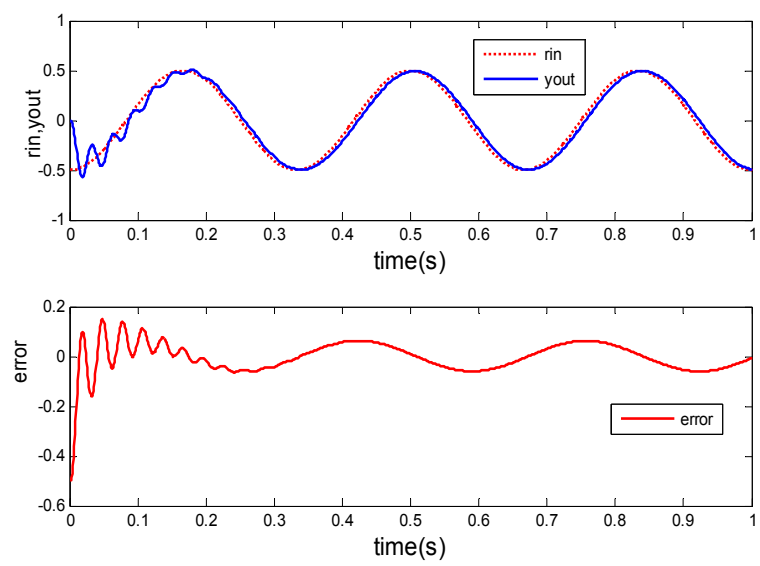

Figure. 6 The results of the common PID control algorithm
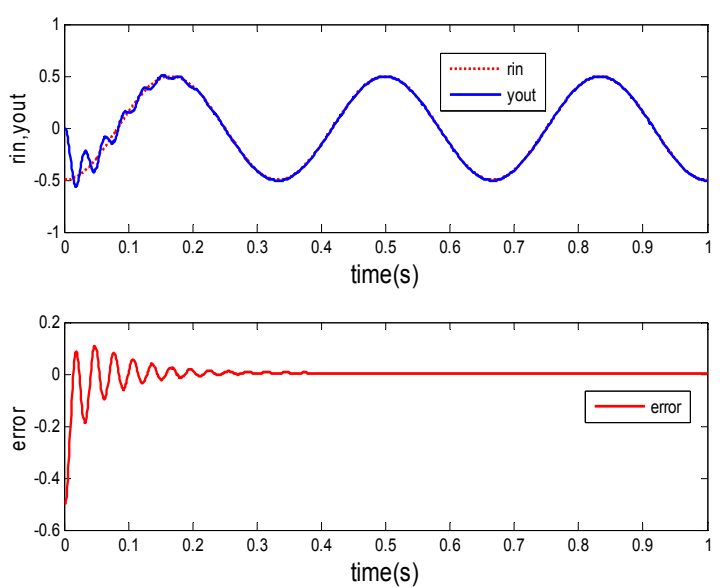

Figure.7 The results of the compounding feedforward PID control algorithm

Figure.6 is the Input/output tracking curves and error change curve of the PID algorithm control. It can be seen the Input/output tracking curves' following performance is not very good. Error change curve is apparently fluctuant, the error fluctuation is \pm 0.1 . Figure. 7 .is the Input/output tracking curves and error change curve of the compounding feedforward PID algorithm control, there is nearly no error between the Input/output tracking curves and this algorithm has good following performance. Error change curve is a less fluctuant at the beginning. After $0.32 \mathrm{~s}$, the error obviously tends to zero. Theatrically, the knitter adopts the compounding feedforward PID control can largely improve its accuracy.

\section{CONCLUSIONS}

This paper analyzes the working principle of shogging of handpiece, adopts the compounding feedforward control to adjust the position error of the shog, improves the accuracy of headpiece's movement, reduces the locating time. This control system has simple structures and low cost, owns a high practical value.

\section{ACKNOWLEDGMENT}

This project is supported by National Natural Science, Fund (No: Y1110686).

\section{REFERENCES}

[1] LI Guo-shan. The structure and technical analysis of importing automatic flat knitting machine, Modern Textile Technology [J], 2002.10, PP24-26.

[2] Song Guang-li. Practical handbook of computerized flat knitter [M], Beijing: China Textile \& Apparel Press, 2010.

[3] Kong Zheng, Jiang Gao-ming and Xia Lin-feng. Investigate on the theory about electronic shogging mechanism of high-speed machines, Knitting Industry[J], 2007.9,PP. 12-14.

[4] Ohishi, K. Hayasaka, E. Nagano, T. etc. Nagano, T. etc. HighPerformance Speed Servo System Considering Voltage Saturation of a Vector-Controlled Induction Motor, IEEE TRANSACTIONS ON INDUSTRIAL ELECTRONICS[J], 2006.6,PP795 - 802. 
[5] Chen Bo-shi, Electric power drag automatic control system[M], Beijing: China Machine Press, 2002.

[6] Liu Jin-kun. Advanced PID control and MATLAB simulation, Beijing: Publishing House of Electronics Industry, 2003.
[7] Grepl, R. Adaptive compounding control of electronic throttle using local learning method,IEEE International Symposium on Industrial Electronics (ISIE)[J], 2010, PP58-61.

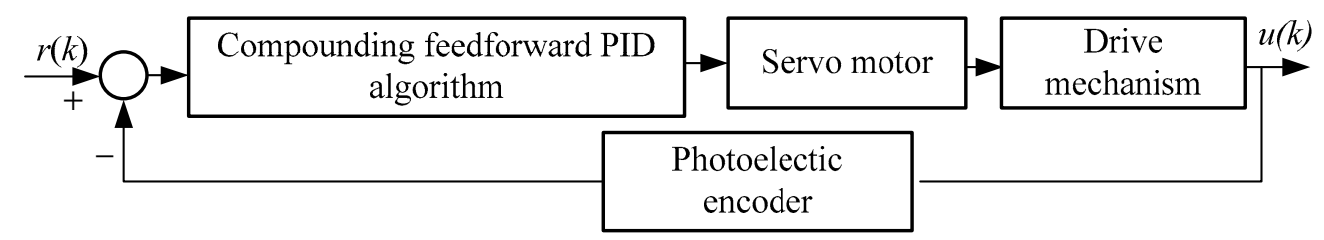

Figure. 2 The block diagram of the shogging closed-loop control

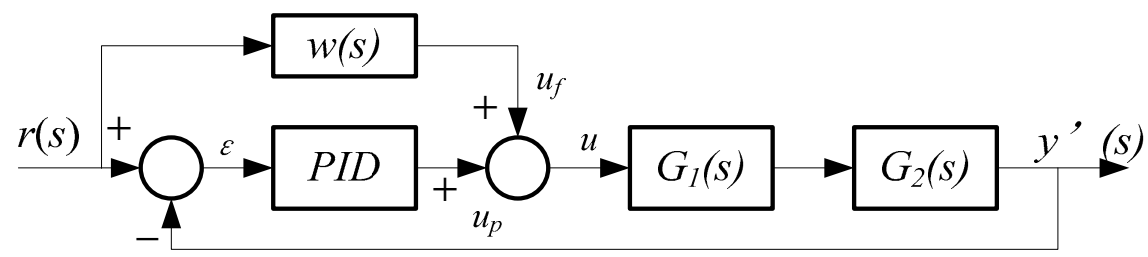

Figure.5 The transfer function's diagram of shogging system 